\title{
Effects of Platycodon grandiflorum methanolic extract on testosterone-induced benign prostatic hyperplasia in Wistar rats
}

\author{
Mee-Young Lee, In-Sik Shin, Nam-Hun Lee, Hyekyung Ha, Jung-Hoon Kim, \\ Chang-Seob Seo and Hyeun-Kyoo Shin*
}

Basic Herbal Research Group, Korea Institute of Oriental Medicine, Exporo 483, Yusung-gu, Daejeon 305-811, Korea.

Accepted 8 May, 2012

\begin{abstract}
Benign prostatic hyperplasia (BPH) is highly prevalent in the male population over the age of 60 years, manifesting as prostatic enlargement and distinctive changes in tissue histomorphology. In this study, we investigated whether a Platycodon grandiflorum methanolic extract (PGME) improved BPH in a testosterone propionate (TP)-induced model of BPH in rats. Castration was performed via the scrotal route under sodium pentobarbital anesthesia, and BPH was induced in the rats with a subcutaneous injection of TP (3 mg/kg) given every consecutive day for 4 weeks after castration. The control group of castrated rats received subcutaneous injections of corn oil. Experimentally, induced rat model of BPH, PGME led to significant reductions in prostate weight and dihydrotestosterone levels in the serum and prostate. Histologically, BPH was evident in the ventral lobe of the prostate, and PGME treatment significantly reduced the severity of the lesion. Therefore, PGME was effective in reducing TP-induced BPH in a rat model, and may be useful for the clinical treatment of patients with BPH.
\end{abstract}

Key words: Benign prostatic hyperplasia (BPH), dihydrotestosterone, testosterone, Platycodon grandiflorum.

\section{INTRODUCTION}

Benign prostatic hyperplasia $(\mathrm{BPH})$ is a multifactorial disease and the commonest noncancerous form of abnormal prostate cell growth affecting older man (Gossel and Wuest, 2004). As the prostate enlarges, it can constrict the urethra, inducing lower urinary tract symptoms (LUTS) such as a weak urinary stream, incomplete bladder emptying, nocturia, dysuria and bladder outlet obstruction (Pais, 2010; Roehrborn, 2011). Currently, the prevalence of BPH is age-dependent, with initial development usually occurring after 40 years of age and as estimated $50 \%$ of men exhibit the symptoms related with $\mathrm{BPH}$ by the age of 50 (Roehrborn and Rosen, 2008).

Although the etiology of BPH is not completely understood, it involves hormonal changes in aging men. The development and growth of the prostate gland

${ }^{*}$ Corresponding author. E-mail: cozy11@kiom.re.kr. Tel: +82-42868-9464. Fax: +82-42-864-2120. depends on androgen stimulation, mainly by dihydrotestosterone (DHT), an active metabolite formed by the enzymatic conversion of testosterone by steroid $5 \alpha$-reductase. According to previous studies, increased conversion of testosterone to DHT with aging, which is catalyzed by prostate $5 \alpha$-reductase, is a crucial alteration in development of BPH. Elevated prostatic DHT level promotes excessive prostatic epithelial and stromal cell growth, causing hyperplasia (Carson and Rittmaster, 2003). So, various drugs have been developed with a focus on reduction of DHT level. Particularly, finasteride, as a $5 \alpha$-reductase inhibitor has been commercially used for treatment of BPH. $5 \alpha$-reductase inhibitors suppressed the development of $\mathrm{BPH}$ via inhibiting the conversion of testosterone to DHT (Foley and Kirby, 2003; Pais, 2010). However, these drugs are limited because of their side effects, including decreased libido, ejaculatory or erectile dysfunction, and nasal congestion (Bullock and Andriole, 2006). To reduce their side effects and reduce the development of $\mathrm{BPH}$, alternative natural materials such 
as saw palmetto and coconut oil have been developed (Bisson et al., 2007; Pais, 2010)

In Asian countries, the root of Platycodon grandiflorum has been widely used as a traditional drug since ancient times. More specifically, it is often prescribed as a folk remedy and herbal medicine for coughs, sputum, asthma, and tonsillitis. Recent studies have shown that materials derived from the Platycodon root are effective against asthma, have anti-inflammatory effects, and act against fatty liver (Ahn et al., 2005; Choi et al., 2009; Khanal et al., 2009). However, no study has examined the efficacy of $P$. grandiflorum on testosterone-induced $\mathrm{BPH}$ in rats. Therefore, in this study, we investigated whether oral dosing with $P$. grandiflorum prevents testosteroneinduced hyperplasia in rats.

\section{MATERIALS AND METHODS}

\section{Preparation of platycodon grandiflorum methanolic extract (PGME)}

Roots of P. grandiflorum were purchased in October, 2008 from HMAX (Chungbuk, Republic of Korea). A voucher specimen has been deposited at the Korea Institute of Oriental Medicine (KIOM), Daejeon, Republic of Korea. The fresh $P$. grandiflorum was washed three times with tap water to remove salts, epiphytes, and sand, and was stored at $-20^{\circ} \mathrm{C}$. The frozen samples were lyophilized and homogenized in a grinder before extraction. The dried roots of $P$. grandiflorum $(300 \mathrm{~g})$ were extracted three times with $70 \% \mathrm{MeOH}(3$ $\mathrm{L})$ with sonication for $1 \mathrm{~h}$. The extract solution was filtered through filter paper and evaporated to dryness $(41.31 \mathrm{~g})$. The yield of the dried extract from the crude starting material was $13.77 \%$. The concentrate was freshly dissolved in phosphate-buffered saline before use.

\section{Animals}

Specific-pathogen-free male Wistar rats, aged 10 weeks, that had been routinely serologically screened for the relevant respiratory pathogens, were purchased from Jung Ang Co. Ltd (Seoul, Korea). The rats were maintained in an animal facility under standard laboratory conditions for 1 week before the experiments, and maintained on a $12 \mathrm{~h}$ light-dark cycle in a room with controlled temperature and humidity. All experimental procedures were carried out in accordance with the NIH Guidelines for the Care and Use of Laboratory Animals, and all animal handling followed the dictates of the National Animal Welfare Law of Korea.

\section{Experimental BPH model and drug administration}

Adult male Wistar rats, weighing 250 to $350 \mathrm{~g}$, were used for the in vivo experiments. After one week of acclimatization, all rats were castrated while anesthetized by an intraperitoneal injection of pentobarbital three days before the beginning of the experiments to exclude the influence of intrinsic testosterone (Sun et al., 2008). Castration was performed by removing the testicles and epididymal fat through the scrotal sac, according to a method published previously (Coppenolle et al., 2000). The spermatic cord and blood vessels were ligated with 3-0 sutures and resected.

$\mathrm{BPH}$ condition was induced by repeated subcutaneous injection of testosterone propionate for 4 weeks according to previous study (Sun et al., 2008; Rick et al., 2011). Experimental groups were divided into 5 groups. Normal control group (A) did not receive any treatment. Castrated rats were randomly divided into four groups (n = 6): (B) castration group, which received PBS administered orally and corn oil injected subcutaneously; (C) BPH group, which received PBS administered orally and testosterone propionate (TP, $3 \mathrm{mg} / \mathrm{kg}$ body weight) injected subcutaneously; (D) PGME group, which received PGME (200 $\mathrm{mg} / \mathrm{kg}$ body weight) administered by oral gavage and TP (3 $\mathrm{mg} / \mathrm{kg}$ body weight) injected subcutaneously; (E) positive control group, which received finasteride (10 mg/kg body weight) administered orally and TP (3 $\mathrm{mg} / \mathrm{kg}$ body weight) injected subcutaneously. The positive control drug, finasteride, is a well-known 5a-reductase inhibitor used for $\mathrm{BPH}$ treatment. All rats were treated once a day for four weeks. Body weight was measured weekly during the experiment. The application volume was calculated in advance, based on the most recent recorded body weight of individual animals.

\section{Blood and tissue samples}

After a treatment period of 4 weeks and an overnight fast, the rats were anesthetized with sodium pentobarbital $(100 \mathrm{mg} / \mathrm{kg}$ body weight, i.p.). Blood samples were collected and centrifuged at 2000 $\times g$ for $10 \mathrm{~min}$. The prostate was collected from each rat and weighed. All prostatic specimens from each group were fixed with $10 \%$ buffered formalin for $24 \mathrm{~h}$. The tissues were then embedded in paraffin, cut into $4 \mu \mathrm{m}$ sections, and stained with hematoxylin (Sigma MHS-16)-eosin (Sigma HT110-1-32) solution. The tissues were then mounted and coverslipped using Dako mounting medium (Dakocytomation, Denmark, CA).

\section{Measurement of DHT and testosterone in prostates and serum}

The prostate tissue was homogenized in lysis buffer containing protease inhibitors (50 mM Tris- $\mathrm{HCl}$ [pH 7.4], $150 \mathrm{mM} \mathrm{NaCl}, 1 \mathrm{mM}$ EDTA, 0.5\% NP-40, 0.1\% SDS, $1 \mathrm{mM}$ EGTA, $100 \mu \mathrm{g} / \mathrm{mL}$ PMSF, 10 $\mu \mathrm{g} / \mathrm{mL}$ pepstatin $\mathrm{A}$, and $100 \mu \mathrm{M} \mathrm{Na} \mathrm{VO}_{3}$ ). The homogenates were centrifuged at $12,000 \times g$ for $25 \mathrm{~min}$ at $4^{\circ} \mathrm{C}$, and the protein concentrations in the supernatant fractions were determined using Bradford reagent (Bio-Rad, Hercules, CA). Enzyme-linked immunosorbent assays (ELISAs) were performed according to the manufacturer's instructions. DHT and testosterone levels in the serum and prostates were measured with ELISA kits (Cayman, USA). The values are expressed per milligram of protein.

\section{Measurement of alanine aminotransferase (ALT) and aspartate aminotransferase (AST) levels in serum}

ALT and AST levels were determined to assess liver and kidney function using commercial kits (Beckman Coulter, Inc., Fullerton, CA, USA) and an autoanalyzer (Beckman CX4, CA, USA).

\section{Image capture and photomicrography}

Photomicrographs were captured with a Photometric Quantix digital camera running a Windows program and montages were assembled in Adobe Photoshop 7.0. The images were cropped and corrected for brightness and contrast, but were not otherwise manipulated.

\section{Statistical analysis}

Data are expressed as means \pm standard deviations (SD). Statistically significant differences were determined with Student's 


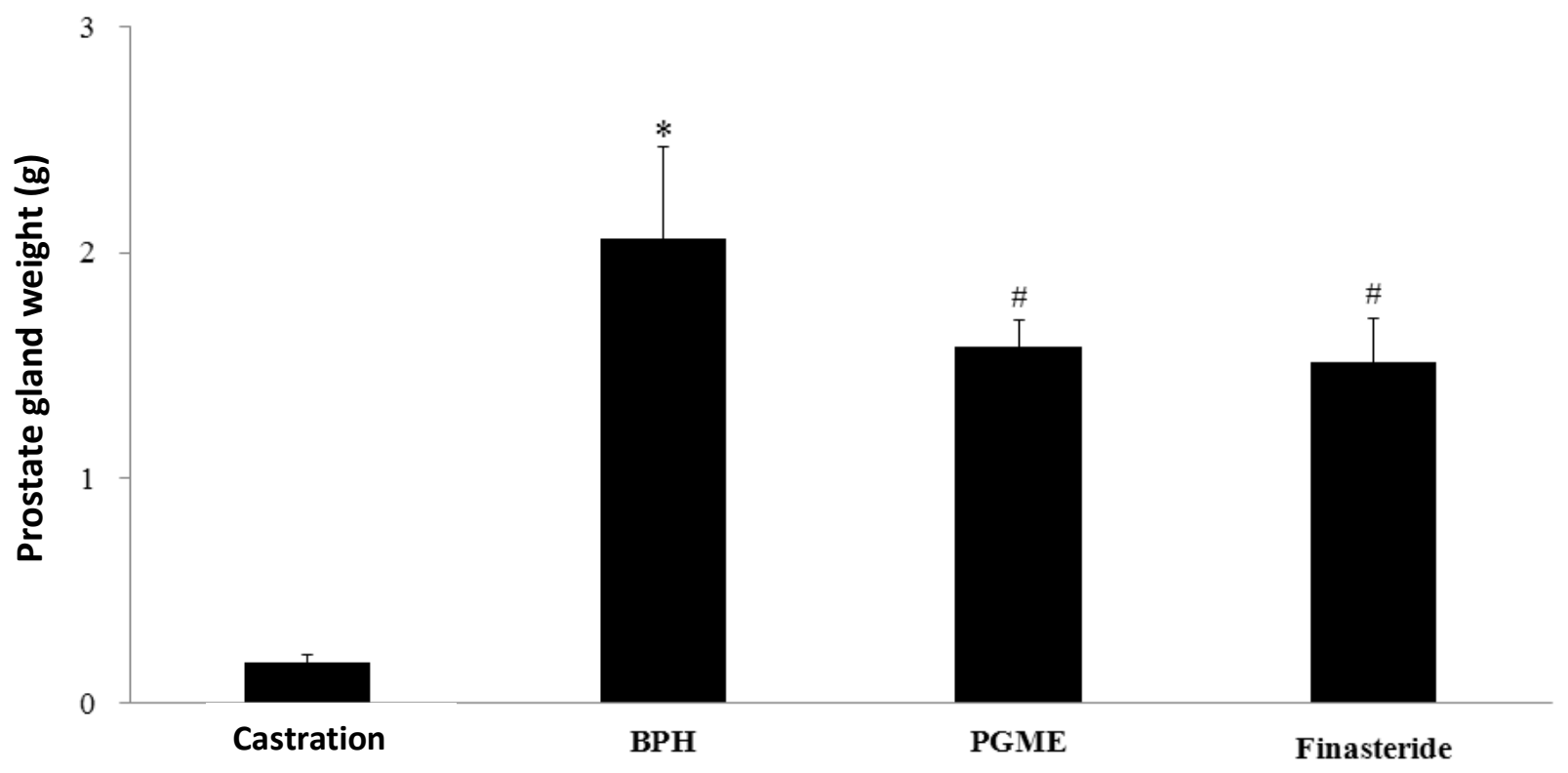

Figure 1. Effects of PGME on prostate weight. The rat model of benign prostate hyperplasia (BPH) was induced with daily injections of testosterone for 4 weeks after castration. BPH group showed a significant increase in the prostate weight compared with castration group. In contrast, PGME group and Finasteride group significantly decreased compared with BPH group. Values are expressed as means \pm SD ( $n=6 /$ group). "Significant difference at $p<0.01$ compared with the castration group. *Significant difference at $p<0.05$ compared with the BPH group.

two-tailed $t$ test when independent means were compared, using Microsoft Excel. The critical level for significance was set at $p<$ 0.05 .

\section{RESULTS}

\section{Effect of PGME on prostate weight}

As shown in Figure 1, the prostate weights of the rats in the various groups were statistically significantly different at the end of the study. The castration group $(0.18 \pm 0.04$ g) markedly decreased the prostate weights when compared with normal control group (1.44 $\pm 0.12 \mathrm{~g})$. In contrast, the BPH group $(2.06 \pm 0.41 \mathrm{~g})$ significantly increased the prostate weight compared with the castration group. The finasteride group $(1.51 \pm 0.20 \mathrm{~g})$ significantly reduced the prostate weights compared with the BPH group. Also, PGME group $(1.58 \pm 0.12 \mathrm{~g})$ was observed the significant reduction in the prostate weight in comparison to the BPH group, which is very similar to the finasteride group.

\section{Effect of PGME on testosterone and DHT levels in serum}

In testosterone levels in serum, there was no significant difference between all groups except for the normal control group (Table 1). In DHT levels in serum, the BPH group statistically increased more than the normal control group $(8.79 \pm 1.20 \mathrm{ng} / \mathrm{mL})$ and castration group $(6.24 \pm$
$1.13 \mathrm{ng} / \mathrm{mL}$ ). Conversely, finasteride group (14.45 \pm 3.62 $\mathrm{ng} / \mathrm{mL}$ ) markedly decreased the DHT levels in serum compared with the BPH group. PGME group (10.07 \pm $2.12 \mathrm{ng} / \mathrm{mL}$ ) also significantly increased the DHT levels in serum as well as the result of finasteride group.

\section{Effect of PGME on DHT levels in prostate}

As shown in Figure 2, the DHT level of BPH group (61.56 $\pm 7.87 \mathrm{ng} / \mathrm{mL}$ ) significantly increased more than the normal control group $(31.96 \pm 0.60 \mathrm{ng} / \mathrm{mL})$ and castration group. In contrast, the finasteride group $(28.23 \pm 6.77$ $\mathrm{ng} / \mathrm{mL}$ ) significantly increased the DHT level in prostate compared with the BPH group. The PGME group (34.77 $\pm 9.70 \mathrm{ng} / \mathrm{mL}$ ) also observed the significant reduction in the DHT level of prostate in comparison to the BPH group, which was similar to the result of finasteride group.

\section{Effect of PGME on the histomorphological changes in prostate tissue}

The prostate of castration group was observed the atrophy of glandular and the reduction of epithelial thickness of prostate compared with the normal control. In contrast, the prostate of BPH group showed the increased thickness of epithelial in prostate caused by epithelial cell hyperplasia. The finasteride group was observed the reduction in epithelial thickness of prostate compared with $\mathrm{BPH}$. The PGME group was observed the 
Table 1. The effects of PGME on DHT and testosterone levels in serum.

\begin{tabular}{lccc}
\hline Group & Dose $(\mathbf{m g} / \mathbf{k g})$ & Testosterone $(\mathbf{p g} / \mathbf{m L})$ & DHT $(\mathbf{n g} / \mathbf{m L})$ \\
\hline NC & - & $133.07 \pm 20.1$ & $8.79 \pm 1.20$ \\
Castration & - & $38.34 \pm 0.3$ & $6.24 \pm 0.50$ \\
BPH & - & $204.04 \pm 39.1 \#$ & $19.16 \pm 3.49^{\#}$ \\
PGME & 100 & $174.99 \pm 31.3$ & $10.58 \pm 2.22^{*}$ \\
Finasteride & 10 & $170.52 \pm 28.1$ & $14.45 \pm 3.61$ \\
\hline
\end{tabular}

${ }^{\#} \mathrm{P}<0.05$ vs. castration group, ${ }^{*} \mathrm{P}<0.05$ vs. $\mathrm{BPH}$ group.

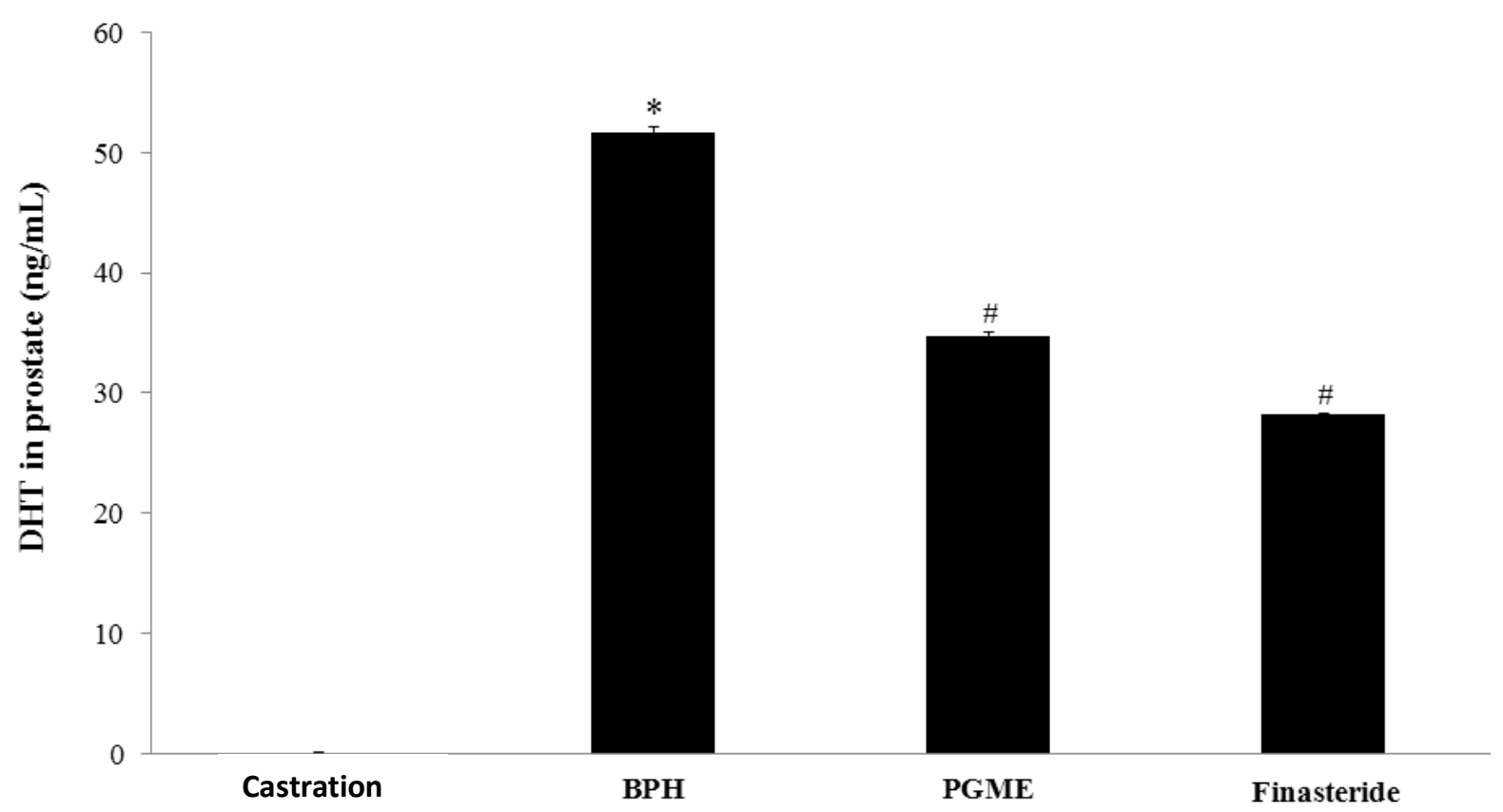

Figure 2. Effects of PGME on DHT levels in prostate. DHT levels in prostate were evaluated using ELISA. BPH group markedly increased DHT level in prostate while PGME and Finasteride groups significantly reduced in comparison to BPH group. Values are expressed as means $\pm S D(n=6 /$ group). "Significant difference at $p<0.01$ compared with the castration group. *Significant difference at $\mathrm{p}<0.05$ compared with the BPH group.

reduction in epithelial thickness of prostate compared with the $\mathrm{BPH}$ group, as well as the finasteride group (Figure 3).

\section{Cytotoxicity of PGME in a BPH model}

PGME did not affect the activities of the serum toxicity marker enzymes, ALT and AST, indicating normal liver and kidney function (Figure 4).

\section{DISCUSSION}

We examined the effects of PGME in a testosteroneinduced rat model of $\mathrm{BPH}$ and compared them with the effects of finasteride, which is currently used to treat $\mathrm{BPH}$.
Treatment with PGME for 4 weeks significantly inhibited the development of testosterone-induced prostatic hyperplasia, which was evident in the reduction in the elevated prostate weight/body weight ratio, reduced DHT levels in the prostate and serum, and histopathological evidence.

The increased prostate weight is used as one of crucial markers of BPH according to previous study (Pais, 2010). $\mathrm{BPH}$ is characterized by stromal and epithelial cells hyperplasia, resulting in prostate enlargement. In previous studies, animals with BPH had a significant increase in prostate weight compared with normal control animals, whereas those of animals treated with finasteride or others herbal remedies for the management of $\mathrm{BPH}$ had meaningfully reduced compared with $\mathrm{BPH}$ animals (Bisson et al., 2007; Pais, 2010). For these reason, many studies have evaluated the inhibitory 


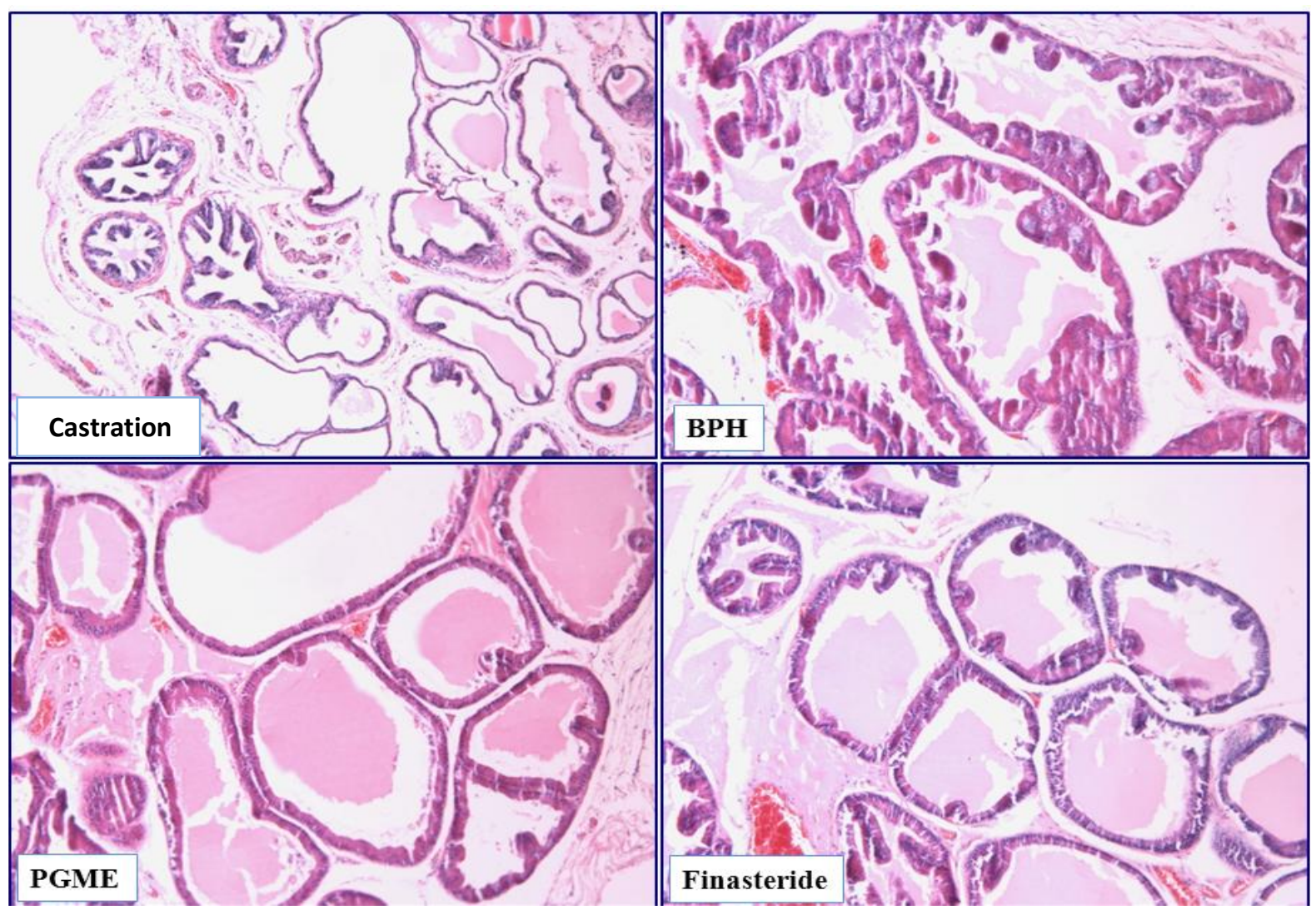

Figure 3. Effects of PGME on prostate hyperplasia. Histological examination of prostate tissue was performed $24 \mathrm{~h}$ after the final testosterone injection. Prostate tissues were fixed, sectioned to $4 \mu \mathrm{m}$, and stained with hematoxylin-eosin solution (magnification $\times 200$ ). BPH group was observed the hyperplasia of epithelial cell. In contrast, PGME and Finasteride groups reduced the hyperplasia of epithelial cell, indicating decrease in epithelial layer thickness when compared with BPH group.

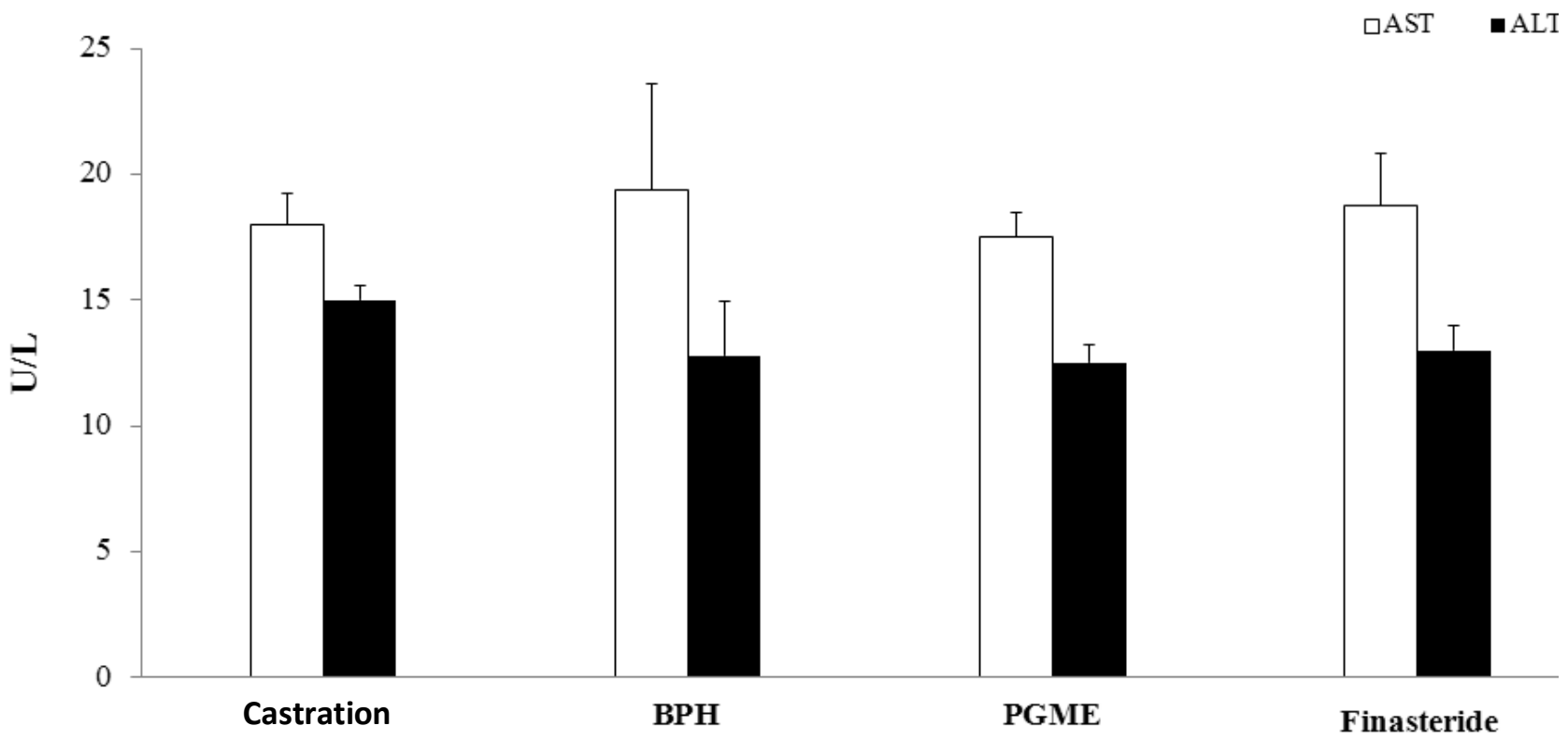

Figure 4. Effects of PGME on serum AST and ALT levels. Serum was collected after the final testosterone injection was given to the rats. Each sample was analyzed using commercial AST/ALT detection kits. There were no significant differences of serum AST and ALT levels between all groups. 
effects of various materials on the development of $\mathrm{BPH}$ by measuring prostate weight (Jang et al., 2010; Veeresh Babu et al., 2010). In present study, the animals with $\mathrm{BPH}$ showed significantly increased prostate weight compared with the normal control animals; however, PGME-treated animals showed the significant reduction in these measures compared with the BPH animals. These findings were consistent histomorphologic examination of prostate. BPH animals showed epithelial hyperplasia with an increase in epithelial thickness compared with the normal control animals. In contrast, PGME-treated animals showed the reduction in epithelial hyperplasia with decrease in epithelial thickness. Based on these results, PGME treatment effectively inhibited the prostatic hyperplasia induced by testosterone.

The two main classes of drugs used as BPH treatments are inhibitors of $\alpha 1$-adrenoceptor inhibitors, which inhibit smooth muscle cell contraction (Furuya et al., 1982), and inhibitors of type II $5 \alpha$-reductase, an enzyme responsible for the conversion of testosterone to the more potent androgen dihydrotestosterone (DHT) (Griffiths and Denis, 2000). Steroid $5 \alpha$-reductase converts testosterone to DHT, an active form of androgen, in the prostate. Increased production of DHT results in the development of prostatic hyperplasia (Pais, 2010). Because DHT has a 10 times higher affinity for the androgen receptor than testosterone, DHT easily binds to androgen receptor, which stimulates the transcription of growth factors that are mitogenic for the epithelial and stromal cells for prostate (Carson and Rittmaster, 2003). The importance of DHT in prostatic hyperplasia was demonstrated by previous studies in which an inhibitor of $5 a$-reductase was administered to experimental animals with $\mathrm{BPH}$ (Roehrborn, 2011). For instance, at clinical doses, finasteride is selective for $5 \alpha$-reductase and achieves about $70 \%$ suppression of serum DHT and $68 \%-86 \%$ suppression of intraprostatic DHT (Span et al., 1999). These findings are in agreement with our results. In present study, PGME reduced the DHT levels in the prostate and serum relative to those in rats with testosterone-induced $\mathrm{BPH}$. Thus, these results indicate that PGME inhibits the development of BPH via downregulation of $\mathrm{DHT}$. Interestingly, although testosterone level in finasteride-treated group was not observed a significant difference, it was decreased compared with the $\mathrm{BPH}$ animals. Before performing present study, we expected the increase in testosterone level according to previous study (Pais, 2010; Roehrborn, 2011). This finding is unlike to previous studies and is unexpected. Many researchers have conducted the studies on relationship between drug treatment and testosterone level in BPH condition. In most previous studies, administration of finasteride showed that the testosterone level is increased compared with that of BPH animals in many studies due to inhibit the transformation testosterone to DHT. On the other hand, some previous studies showed that the testosterone level is similar to that of $\mathrm{BPH}$ animals following drug treatment as well as the result of present study (Gasco et al., 2007). However, we did not find the clear reason why testosterone level is not changed in present study.

\section{Conclusion}

Oral administration of PGME in a $\mathrm{BPH}$ rat model significantly decreased the prostate weight, prostatic epithelial hyperplasia, and DHT levels in the serum and prostate. These results indicate that PGME may effectively inhibit the development of BPH. A further study is required to explore the effects of the major active components of Platycodon grandiflorum and the molecular mechanisms underlying their action.

\section{ACKNOWLEDGEMENT}

This research was supported by a grant from 'The Evidence-based Medicine for Herbal Formulae' from the Korea Institute of Oriental Medicine (KIOM).

\section{REFERENCES}

Ahn KS, Noh EJ, Zhao HL, Jung SH, Kang SS, Kim YS (2005). Inhibition of inducible nitric oxide synthase and cyclooxygenase II by Platycodon grandiflorum saponins via suppression of nuclear factorkappaB activation in RAW 264.7 cells. Life Sci. 76:2315-2328.

Bisson JF, Hidalgo S, Rozan P, Messaoudi M (2007). Therapeutic effect of ACTICOA powder, a cocoa polyphenolic extract on experimentally induced prostate hyperplasia in Wistar-Unilever rats. J. Med. Food 10:628-635.

Bullock TL, Andriole GL (2006). Emerging drug therapies for benign prostatic hyperplasia. Expert Opin. Emerg. Drugs 11:111-123.

Carson C, Rittmaster R (2003). The role of dihydrotestosterone in benign prostatic hyperplasia. Urology 61(1):2-7.

Choi JH, Hwang YP, Lee HS, Jeong HG (2009). Inhibitory effect of Platycodi Radix on ovalbumin-induced airway inflammation in a murine model of asthma. Food Chem. Toxicol. 47:1272-1279.

Coppenolle FV, Bourhis X, Carpentier F, Delaby G, Cousse H, Raynaud JP, Prevarskaya N (2000). Pharmacological effects of the lipidosterolic extract fo Serenoa repens (Permixon) on rat prostate hyperplasia induced by hyperprolactinemia: comparison with finasteride. Prostate 43:49-58.

Foley CL, Kirby RS (2003). 5 alpha-reductase inhibitors: What's New?. Current. Opinion. In. Urology 13:31-37.

Furuya S, Kumamoto Y, Yokoyama E, Tsukamoto T, Lzumi T, Abiko Y (1982). Alphaadrenergic activity and urethral pressure in prostatic zone in benign prostatic hypertrophy. J. Urol. 128:836-839.

Gossel TA, Wuest JR (2004). New drugs of 2004 for treatment of benign prostatic hyperplasia: Avodart and Uroxatal. Contin. Educ. Pharm. 22:1-4.

Griffiths K, Denis LJ (2000). Exploitable mechanisms for the blockade of androgenic action. Prostate 10:43-51.

Gasco M, Villegas L, Yucra S, Rubio J, Gonzales CF (2007). Doseresponse effect of Red Maca (Lepidium myenii) on benign prostatic hyperplasia induced by testosterone enanthate. Phytomedicine 14:460-464.

Jang H, Ha US, Kim SJ, Yoon BI, Han DS, Yuk SM, Kim SW (2010). Anthocyanin extracted from black soybean reduces prostate weight and promotes apoptosis in the prostatic hyperplasia-induced rat model. J. Agric. Food Chem. 58:12686-12691.

Khanal T, Choi JH, Hwang YP, Chung YC, Jeong HG (2009). Protective 
effects of saponins from the root of Platycodon grandiflorum against fatty liver in chronic ethanol feeding via the activation of AMP. dependent protein kinase. Food Chem. Toxicol. 47:2749-2754.

Pais P (2010). Potency of a novel saw palmetto extract, SPET-085, for inhibition of 5alpha-reductase II. Adv. Ther. 27:555-563.

Rick FG, Schally AV, Block NL, Halmos G, Perez R, Fernandez JB, Vidaurre I, Szalontay L (2011). LHRH antagonist Cetrorelix reduces prostate size and gene expression of proinflammation cytokines and growth factors in a rat model of benign prostatic hyperplasia. Prostate 71:736-747.

Roehrborn CG (2011). Male lower urinary tract symptoms (LUTS) and benign prostatic hyperplasia (BPH). Med. Clin. North Am. 95: 87-100.

Roehrborn CG, Rosen RC (2008). Medical therapy options for aging men with benign prostatic hyperplasia: focus on alfusozin $10 \mathrm{mg}$ once daily. Clin. Interv. Aging 3:511-524.

Span PN, Völler MC, Smals AG, Sweep FG, Schalken JA, Feneley MR, Kirby RS (1999). Selectivity of finasteride as an in vivo inhibitor of 5alpha-reductase isozyme enzymatic activity in the human prostate. J. Urol. 161:332-337.
Sun H, Li TJ, Sun LN, Qiu Y, Huang BB, Yi B, Chen WS (2008). Inhibitory effect of traditional Chinese medicine Zi-Shen Pill on benign prostatic hyperplasia in rats. J. Ethonopharmacol. 115:203-208.

Veeresh BSV, Veeresh B, Patill AA, Warke YB (2010). Lauric acid and myristic acid prevent testosterone induced prostatic hyperplasia in rats. Eur. J. Pharmacol. 625:262-265. 